\title{
sciendo
}

10.2478/AMB-2019-0018

\section{NON-SURGICAL CORRECTION OF NASAL DORSUM AND TIP IN PRIMARY CASES}

\author{
Y. P. Yordanov ${ }^{1,2}$, A. Shef ${ }^{2}$ \\ ${ }^{1}$ Adella Aesthetic - Sofia, Bulgaria \\ ${ }^{2}$ ASD Consult - Sofia, Bulgaria
}

\begin{abstract}
Nowadays, minimally invasive and non-invasive approaches in aesthetic medicine are increasingly popular and non-surgical rhinoplasty is one of the procedures with rising demand worldwide. In Bulgaria, this procedure seems to be uncommonly performed, since there are no reports in the local scientific literature. The aim of this preliminary study was to briefly explore the possibilities of hyaluronic acid (HA) fillers for correcting three of the most common nasal deformities - dorsal hump, under-projected and under-rotated nasal tip in primary cases. In order to accomplish this objective, a retrospective clinical study was conducted encompassing 11 cases of the authors' practices with the above mentioned nasal irregularities and no previous surgical/non-surgical treatment. The analysis of patient-reported outcomes suggested a high satisfaction rate, with no major complication in the series. The HA filler has shown to be safe, relatively easily applicable and highly effective for camouflaging nasal deformities. A special emphasis was put on the patient safety and ten golden rules for staying out of trouble were given.
\end{abstract}

Key words: hyaluronic acid filler, rhinoplasty, non-surgical treatment, patient safety

Corresponding author: Yordan P. Yordanov MD, PhD, FEBOPRAS, Adella Aesthetic; 15G, Tintyava Str., 1172 Sofia, Bulgaria, e-mail: yordanov_vma@abv.bg

\section{INTRODUCTION}

A ccording to the International Society of Aesthetic Plastic Surgery (ISAPS) annual Global Aesthetic Survey on procedures performed in 2017 , rhinoplasty is one of the five fastest growing aesthetic procedures worldwide [1] and surgical rhinoplasty is the golden standard in nasal correction in terms of aesthetics and function [2, 3]. However, surgical approach has several disadvantages, including the need of general anaesthesia, hospital stay, splints, postopertative swelling and bruising, downtime and risk of late complications, such as irreversible asymmetry and functional impairment. On the other hand, the use of HA fillers for various aesthetic applications is increasingly popular and demographic data have shown an expanding treatment-requiring population over the last years [4]. Therefore, it seems comprehensible why non-surgical nasal correction with $\mathrm{HA}$ is becoming more popular nowadays.

The aim of this preliminary study was to explore the possibilities of HA fillers for correcting three of the most common nasal deformities in the authors' practices: dorsal hump, under-projected and underrotated nasal tip.

\section{MATERIAL AND METHODS}

A retrospective analysis based on the clinical records and photographs of patients with dorsal hump defor- 
mity and under-projected and under-rotated nasal tip, treated in the authors' practices, was performed for a period of two years. Patient demographic and medical data were collected, including age, common habits, comorbidities, current and past therapy, history of previous treatments, herpes simplex or other skin infection. The following were selected as exclusion criteria: history of any previous surgical/non-surgical nasal correction, local infection, connective tissue disease, congenital or iatrogenic bleeding disorder, hypersensitivity to lidocaine or HA, current therapy with coagulation modifiers, malignant lesions in the treated area.

Both written and verbal informed consent was obtained from each patient for the injection procedure, possible risks and complications, and utilization of the patient's personal data and photographs. The same treatment protocol was applied for all cases in the ambulatory settings: skin was disinfected with $70 \%$ alcohol solution and the same HA filler was used. The product of choice was Restylane (Q-Med, Uppsala, Sweden) - a high-G' product $(512 \mathrm{~Pa})$ with $\mathrm{HA}$ concentration of $20 \mathrm{mg} / \mathrm{ml}$ and lidocaine hydrochloride of $3 \mathrm{mg} / \mathrm{ml}$. A 25-gauge blunt cannula and/or 27-gauge needle was used according to the injector's preference and individual patient needs.

For correcting the nasal dorsum, the procedure started with an injection of the glabella and then continued caudally, in order to extend and define the nasofrontal angle. Augmentation of the nasal dorsum below the hump was performed and was continued toward the nasal tip when indicated.

When dealing with the tip, an injection deep to the anterior nasal spine and nasal base was performed first, in order to provide support to and open the nasolabial angle producing a push-up effect on the columella. When additional projection and cephalic rotation was required, a nasal tip injection was done. In the case of an aesthetically unpleasant supratip break, a HA ap- plication between the domes was carried out. Topical ice after injection was applied for 20 minutes.

The protocol for the minimum 12-month follow-up of all patients included clinical examinations by the responsible injector, carried out on Day 10 and by the end of 1, 3, 6 and 12 months. A photograph for each of the five standard views (frontal, lateral, 45-degreeangle, worm's-eye and above view) was taken before injection and by the end of 1, 6 and 12 months. The photographs were evaluated independently by the two authors. A touch-up with additional injection of HA filler was done on Day 10, when needed.

\section{RESULTS}

A total of 11 patients, aged between 27 and 42 years (mean age 33.82 years) (Table 1) were treated for the selected study period. Out of them, eight were female $(n=8 ; 72.7 \%)$ and three were male $(n=3 ; 27.3 \%)$. In all the patients, dorsal hump deformity and under rotation of the tip were presented. In 4 of them, supratip or under projection of the tip was registered too. In the series, the mean total volume of the filler for nonsurgical correction was $1.2 \mathrm{ml}$ for the female patients and $1.7 \mathrm{ml}$ for the male patients. Three patients $(\mathrm{n}=$ $3 ; 27.3 \%$ ) required a touch-up on Day 10 because of subjective undercorrection, 2 of them were male.

Mild pain and increased sensitivity were registered in two patients $(\mathrm{n}=2 ; 18.2 \%$ ) on the first post-procedural day, which subsided spontaneously within 3 days. Bruising was observed in three patients $(n=3$; $27.3 \%$ ) and Arnica-containing cream was prescribed with rapid resolution of the condition. No case of haematoma, skin necrosis or skin loss was observed.

Good aesthetic outcomes and high patients' satisfaction were assessed and registered on the follow-up examinations in 6 and 12 months (Figure 1).

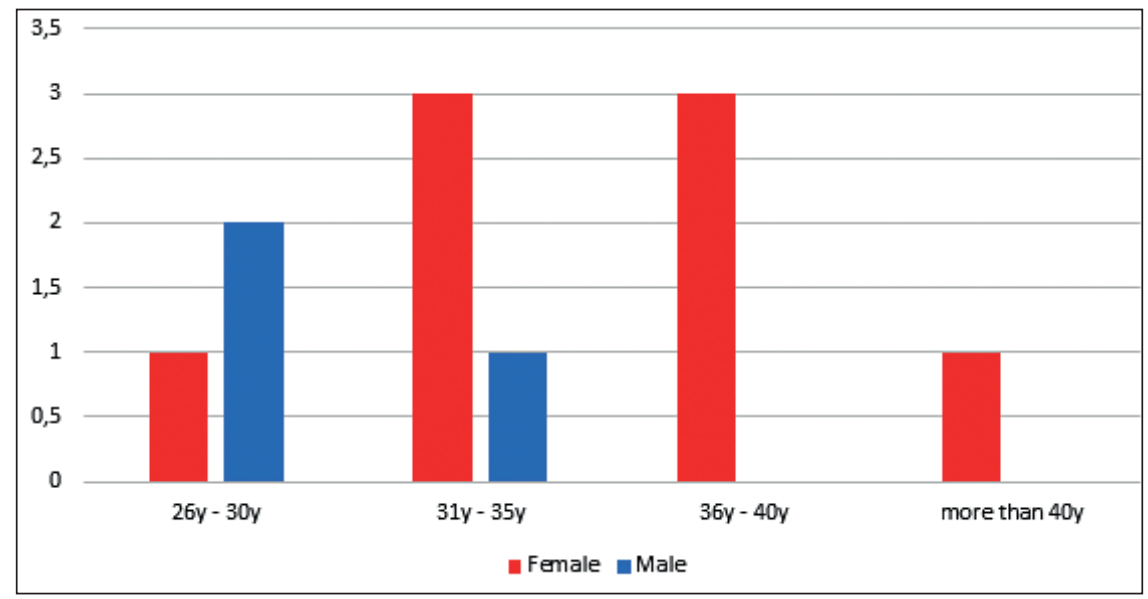

Table 1. Distribution of the patients by sex and age at the time of the first treatment 

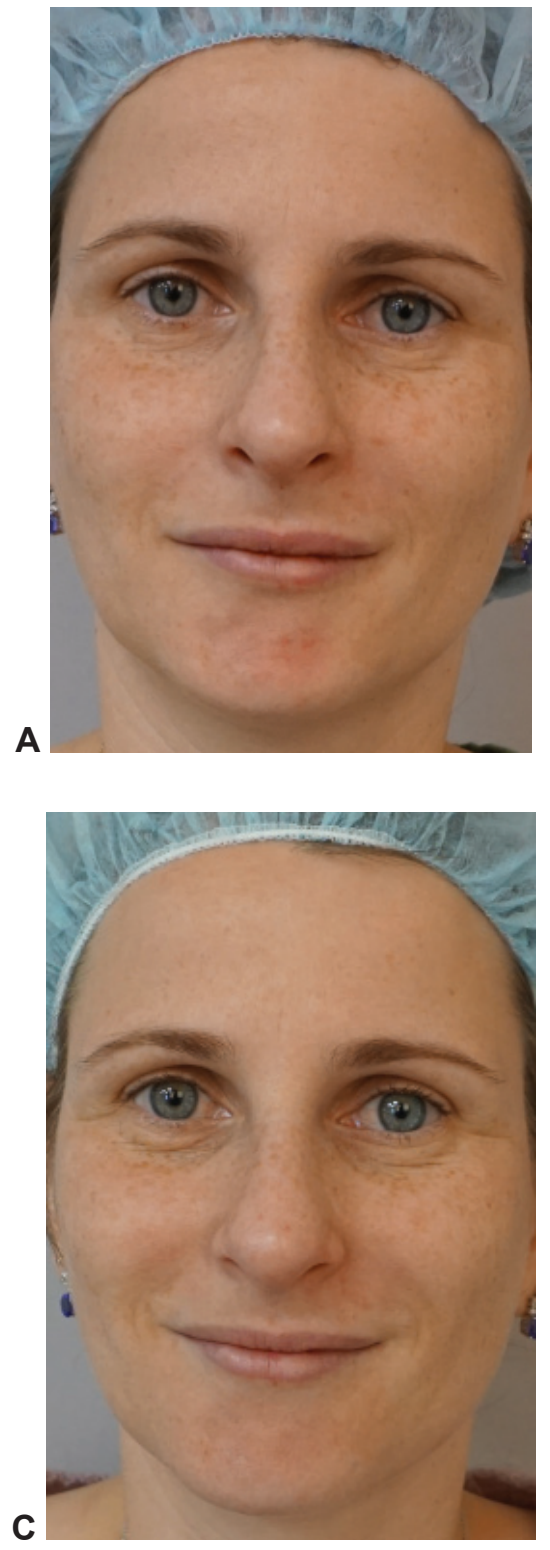
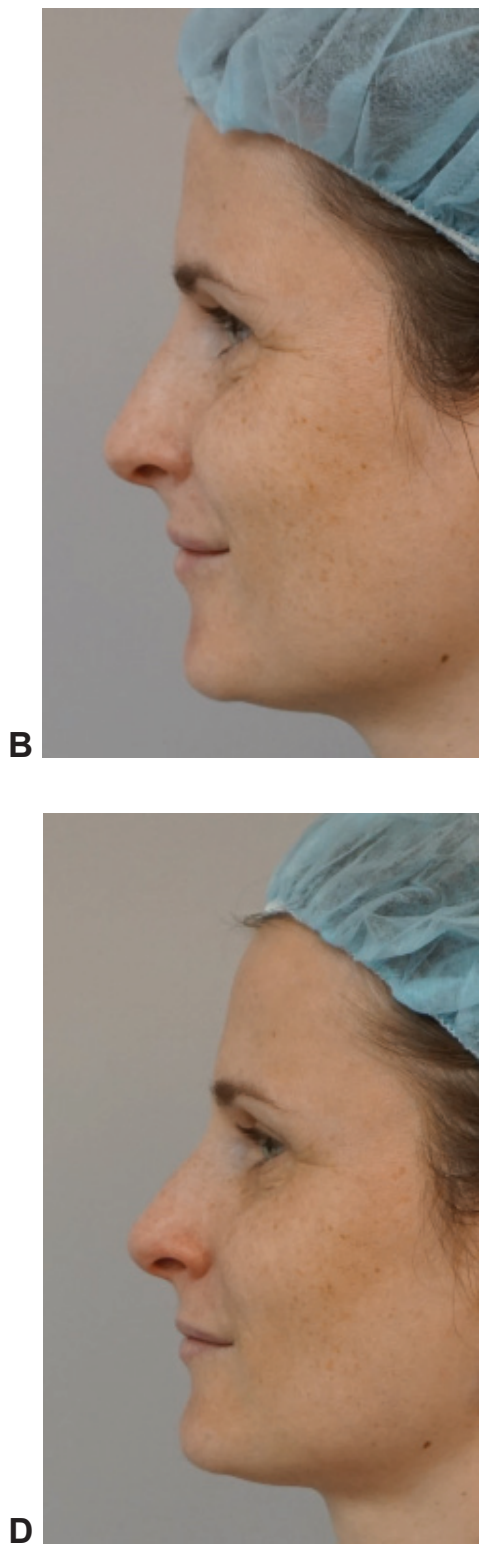

Fig. 1. (A, B) A 34-year-old primary female patient with a slight deformity of the dorsal aesthetic lines, minimal dorsal hump and under-projected and under-rotated nasal tip; (C, D) Follow-up examination by the end of 12 months after a single-stage injection of $1.2 \mathrm{ml}$ Restylane was applied into the nasal dorsum, tip and columella (deep to the nasal spine): greatly improved dorsal aesthetic lines (C), camouflaged dorsal hump, increased tip projection and gentle tip rotation were observed (D)

\section{DISCUSSION}

Nowadays, the demand for minimally invasive or non-invasive procedures in aesthetic medicine is increasingly growing. HA fillers have shown to be a wonderful solution for a great variety of age-related changes or just for beautification purposes [4, 5]. Non-surgical rhinoplasty is becoming more and more popular procedure worldwide. Nevertheless, in Bulgaria it is still not so commonly performed and so far, there are no scientific reports on this topic. Therefore, we are convinced that this procedure re- mains not well known among the Bulgarian practitioners in terms or possibilities and safety profile. Because of the above, the focus of our preliminary work was directed to correction of the most common deformities particularly in primary cases.

Talking about good medical practice, an extremely important issue is patient safety, which always should be a must for every professional performing aesthetic procedures. When applying HA filler in particular, one should always keep in mind the following 10 golden rules for nasal injection, to avoid any complications: 
1. Asepsis is of paramount importance. Perform meticulous skin disinfection before and during the treatment. Work with gloves, clean the gloves and change them every time when needed;

2. When injecting, stay on the midline as much as possible. The main nasal blood vessels normally lie away from the midline - that is why it is a safe plan of injection. As variations in the anatomy do exist, they could place some patients at a higher risk of intravascular injection. It has been shown that in some individuals dorsal nasal vessels cross the midline, which tends to happen usually at the middle third of the nose $[2,6]$ - stay alert when working in this area;

3. Aspirate for a few seconds before starting injection;

4. Use a 27-gauge or wider blunt cannula or needle;

5. Use a retrograde technique of injection;

6. Stay in the right plan:

- in the glabella, nasal dorsum, anterior nasal spine and columella, the injection is deep to the periosteum, i.e. supraperiosteal injection;

- into the nasal ala and the tip, the injection can be deep dermal. This plan could also be used in the glabella area, when needed [7];

7. Inject slowly and use small volumes of the material;

8. Stay attend and always observe the patient for signs of vascular compromise during the injection;

9. Pinch the soft tissue of the treated area in the midline, to avoid lateral spreading of the filler.

10. Spread the HA filler with gentle smoothing massage at the end of every injection with wet gloves, to avoid "bumps and lumps".

Having in mind the above rules, we can apply the non-surgical approach for camouflaging nasal deformities. Although from a small series, our results are comparable with those of Bertossi at al. [2] and other authors $[8,9]$ in terms of complications and outcomes. Nevertheless, our main concern is always vascular compromise, especially when working in the glabella area, which has been suggested to be the most common site of the most devastating complication in filler injection practice - blindness [10]. In addition to the higher vascularity of the glabellar and entire perinasal area, in secondary cases the injec- tor should be aware of possible changes in the topography and quality of vessels due to the previous surgery $[11,12]$.

\section{CONCLUSION}

In this brief clinical study, the application of Restylane hyaluronic acid filler has shown to be safe, relatively easily applicable and highly effective for camouflaging nasal deformities, such as dorsal and tip irregularities. Special attention should be paid to patient safety at the time of applying fillers in the nasal and perinasal area.

\section{REFERENCES}

1. Global Aesthetic Survey. ISAPS 2017. https://www.isaps.org/ wp-content/uploads/2018/10/2017-Global-Survey-Press-Release-Demand-for-Cosmetic-Surgery-Procedures-AroundThe-World-Continues-To-Skyrocket_2_RW.pdf

2. Bertossi D, Lanaro L, Dorelan $S$, et al. Nonsurgical Rhinoplasty: Nasal Grid Analysis and Nasal Injecting Protocol. Plast Reconstr Surg. 2019;143(2):428-39.

3. Cosmetic surgery national data bank statistics. Aesthet Surg J. 2015;35(Suppl 2):1-24.

4. Sundaram H, Liew S, Signorini M, et al. Global Aesthetics Consensus: Hyaluronic Acid Fillers and Botulinum Toxin Type A-Recommendations for Combined Treatment and Optimizing Outcomes in Diverse Patient Populations. Plast Reconstr Surg. 2016;137(5):1410-23.

5. Yordanov YP, Shef A. Nonsurgical rejuvenation of the middle third of the face with hyaluronic acid fillers. Medical Review 2017;53(3):43-8

6. Tansatit T, Apinuntrum P, Phetudom T. Facing the Worst Risk: Confronting the Dorsal Nasal Artery, Implication for Nonsurgical Procedures of Nasal Augmentation. Aesthetic Plast Surg. 2017;41(1):191-8.

7. Humphrey CD, Arkins JP, Dayan SH. Soft tissue fillers in the nose. Aesthet Surg J. 2009;29(6):477-84.

8. Schanz S, Schippert W, Ulmer A, et al. Arterial embolization caused by injection of hyaluronic acid (Restylane). Br J Dermatol. 2002;146(5):928-9.

9. Urdiales-Gálvez F, Delgado NE, Figueiredo V et al. Treatment of Soft Tissue Filler Complications: Expert Consensus Recommendations. Aesthetic Plast Surg. 2018;42(2):498-510.

10. Beleznay K, Carruthers JDA, Humphrey S, et al. Update on Avoiding and Treating Blindness from Fillers: A Recent Review of the World Literature. Aesthet Surg J. 2019 Feb 21. pii: sjz053. doi: 10.1093/asj/sjz053.

11. Hedén P. Nasal Reshaping with Hyaluronic Acid: An Alternative or Complement to Surgery. Plast Reconstr Surg Glob Open. 2016;4(11):e1120.

12. Liapakis IE, Englander M, Vrentzos NP, et al. Secondary rhinoplasty fixations with hyaluronic acid. J Cosmet Dermatol. 2013;12(3):235-9. 